\title{
Existence of Phase Transitions for Quantum Lattice Systems
}

\author{
J. GiNiBRE \\ Laboratoire de Physique Théorique et Hautes Energies, Orsay, France`
}

Received July 7, 1969

\begin{abstract}
We prove that the following lattice systems:
(1) anisotropic Heisenberg model,

(2) Ising model with transverse magnetic field,

(3) quantum lattice gas with hard cores extending over nearest neighbours, exhibit phase transitions if the temperature is sufficiently low and the transverse (or kinetic) part of the interaction sufficiently small.
\end{abstract}

\section{Introduction}

The existence of a phase transition at sufficiently low temperature has been proved for a variety of Ising models with attractive interactions [1-7] on $v$-dimensional cubic lattices $(v \geqq 2)$. The argument goes back to Peierls [1]. It can be described in the lattice gas language, and rests on the following ingredients:

(1) Probability estimate. With each configuration on the lattice, one associates a family of closed polygonal or polyhedral contours. Let $G$ be such a contour, and $g$ the area of its boundary. One first proves that the probability of occurrence of a given $G$ is bounded by

$$
P(G) \leqq \exp [-\beta a g]
$$

where $\beta$ is the inverse temperature and $a$ some positive constant.

(2) Entropy estimate. The number of possible shapes of $G$ for a given $g$ is bounded by $3^{g-v}$.

(3) Density estimate. For a given $g$, the volume enclosed in $G$ is bounded by $(g / 2 v)^{v / v-1}$, corresponding to the worst possible shape, which is a cube.

From these estimates and general arguments, one deduces the existence of at least two equilibrium states corresponding to the same temperature and chemical potential (or magnetic field), with densities

\footnotetext{
* Laboratoire associé au Centre National de la Recherche Scientifique.
} 
$\varrho \leqq \varrho_{0}<\frac{1}{2}$ and $\varrho^{\prime} \geqq 1-\varrho_{0}>\frac{1}{2}$, where

$$
\varrho_{0}=\sum_{g} 3^{-v}\left(\frac{g}{2 v}\right)^{\frac{v}{v-1}} 3^{g} \exp [-\beta a g] .
$$

The sum runs over all even integers $g \geqq 2 v$, and the RHS obviously becomes less than $1 / 2$ for $\beta$ sufficiently large. The transition thereby obtained is of first order.

More recently [5], Dobrushin has extended the proof to the case of the Ising model with repulsive interaction, and to lattice gas models with extended hard cores. No claim is made concerning the order of the transition.

In the present paper, we extend the proof to a number of quantum lattice systems in $v \geqq 2$ dimensions, obtained by adding to the classical Hamiltonian either a transverse (i.e. kinetic) interaction term, or a transverse magnetic field. More precisely, we prove that if the temperature is sufficiently low and the admixture of transverse terms sufficiently small, the latter do not destroy the longitudinal spontaneous magnetization (or sub-lattice magnetization) which arises in the corresponding classical system. The proof requires one more step, which precedes the previous ones:

(0) Using Trotter's formula, one reduces the problem of dealing with non commuting operators to a geometrical problem of the same type as the classical one, at the expense of increasing the dimension from $v$ to $(v+1)$.

In Section 2, we give a general characterization of phase transitions which makes clear what has to be proved. In Section 3, we describe the models which we consider, we apply Trotter's formula [8] to reduce the problem to one of the classical type, and introduce the geometrical definitions needed for the proofs. We then consider specific models (Sections 4 to 8 ) in the following order, which is that of increasing difficulty:

(1) Quantum system with extended hard cores,

(2) Anisotropic Heisenberg ferromagnet,

(3) System with extended hard core and transverse magnetic field,

(4a) Ising ferromagnet with transverse magnetic field,

(4b) Ising antiferromagnet with transverse magnetic field,

(5) Anisotropic Heisenberg antiferromagnet.

If the dimension is $v=2$, it is known [9] that:

(a) the anisotropic Heisenberg model does not exhibit transverse spontaneous magnetization.

(b) the isotropic Heisenberg model does not exhibit spontaneous magnetization. 
Our proof in the corresponding case lies far outside of the reach of these results, since we are looking for longitudinal magnetization with only a small admixture of transverse interaction.

\section{Characterization of a Phase Transition}

Consider a quantum system in a box $\Lambda$ with volume $V$. Let $H_{A}$ be the hamiltonian and $A_{A}$ a physical observable. We take $\beta=1$ and disregard all the problems associated with unbounded operators, which are irrelevant for our purpose. Consider the quantity:

$$
p_{\Lambda}(\alpha)=V^{-1} \log \operatorname{Tr} \exp \left[-H_{\Lambda}+\alpha V A_{\Lambda}\right] .
$$

Then $p_{A}(\alpha)$ is a convex function of $\alpha$, as follows from the explicit expression of its second derivative, or from an argument of Peierls (see, for instance, Ref. [11]). Suppose that when $\Lambda$ becomes infinite in a suitable sense, the following limit exists:

$$
p(\alpha)=\lim _{\Lambda \rightarrow \infty} p_{\Lambda}(\alpha)
$$

In practice, $A_{A}$ will be the magnetization, or the sublattice magnetization. For all the systems we shall consider, the limit (2.2) will exist. The function $p(\alpha)$ is then a convex function of $\alpha$. Let $p_{-}^{\prime}(\alpha)$ and $p_{+}^{\prime}(\alpha)$ be the left and right derivatives at $\alpha .\left(p_{-}^{\prime}(\alpha) \leqq p_{+}^{\prime}(\alpha)\right)$. If $p_{-}^{\prime}(\alpha)=p_{+}^{\prime}(\alpha)$, then:

$$
p_{-}^{\prime}(\alpha)=p_{+}^{\prime}(\alpha)=\lim _{\Lambda \rightarrow \infty} p_{\Lambda}^{\prime}(\alpha) .
$$

We shall say that the system exhibits a phase transition if for suitably chosen $A_{\Lambda}$, one obtains $p_{+}^{\prime}(0)-p_{-}^{\prime}(0)>0$. The physical interpretation is clear. We now give a sufficient condition for the existence of a phase transition in this sense. We shall use the following result:

Lemma 1. Let $H_{A}$ and $A_{A}$ be such that the limit (2.2) exists. Suppose that for each $\Lambda$, there is defined a projection operator $P_{A}$ in the Hilbert space associated with $\Lambda$, satisfying the following property: let:

$$
p_{P_{A}}(\alpha)=V^{-1} \log \operatorname{Tr} P_{A} \exp \left[\left(-H_{A}+\alpha V A_{A}\right) P_{A}\right]
$$

and suppose that when $\Lambda$ becomes infinite:

$$
\begin{aligned}
& \text { (a) } p_{P_{\Lambda}}(0) \rightarrow p(0) \\
& \text { (b) } \varlimsup_{\Lambda \rightarrow \infty}\left\langle A_{\Lambda}\right\rangle_{P_{\Lambda}} \leqq \lambda
\end{aligned}
$$

where $\lambda$ is some real number, and $\langle.\rangle_{P_{A}}$ is the expectation value taken with the density matrix $P_{A} \exp \left(-P_{A} H_{A}\right)$.

Then:

$$
p_{-}^{\prime}(0) \leqq \lambda \text {. }
$$


Proof. Let $\alpha>0$;

$$
\begin{aligned}
p_{\Lambda}(0)-p_{A}(-\alpha) & =\left(p_{A}(0)-p_{P_{A}}(0)\right) \\
& +\left(p_{P_{A}}(0)-p_{P_{A}}(-\alpha)\right)+\left(p_{P_{A}}(-\alpha)-p_{A}(-\alpha)\right) .
\end{aligned}
$$

Now $p_{P}(\alpha)$ is a convex function of $\alpha$ and an increasing function of $P$. Therefore the second term in the RHS of (2.8) is less than $\left\langle A_{A}\right\rangle_{P_{A}}$, and the third term is negative. Therefore:

$$
p_{\Lambda}(0)-p_{\Lambda}(-\alpha) \leqq p_{\Lambda}(0)-p_{P_{\Lambda}}(0)+\alpha\left\langle A_{\Lambda}\right\rangle_{P_{A}} .
$$

Taking the limit $\Lambda \rightarrow \infty$, we obtain:

$$
p(0)-p(-\alpha) \leqq \alpha \lambda .
$$

Taking the limit $\alpha \rightarrow 0$, we obtain (2.7).

We can now formulate a sufficient condition for the existence of a phase transition:

Lemma 2. Let $H_{A}$ and $A_{A}$ be as above. Suppose that for each $\Lambda$, there are defined two projectors $P_{A}$ and $Q_{A}$, such that for some real numbers $\lambda$ and $\mu(\lambda<\mu)$ :

$$
\begin{aligned}
& \text { (a) } \lim _{\Lambda \rightarrow \infty} p_{P_{\Lambda}}(0)=\lim _{\Lambda \rightarrow \infty} p_{Q_{\Lambda}}(0)=p(0) \\
& \text { (b) } \lim _{\Lambda \rightarrow \infty}\left\langle A_{\Lambda}\right\rangle_{P_{A}} \leqq \lambda<\mu \leqq \lim _{\Lambda \rightarrow \infty}\left\langle A_{\Lambda}\right\rangle_{Q_{A}}
\end{aligned}
$$

Then the system exhibits a phase transition in the previous sense.

Proof. From Lemma 1, we obtain

$$
p_{-}^{\prime}(0) \leqq \lambda<\mu \leqq p_{+}^{\prime}(0)
$$

which proves Lemma 2.

In practice, $P_{A}$ and $Q_{A}$ will be taken from the classical proof in an obvious way, (2.11) will also be obvious, and we shall concentrate on proving (2.12).

\section{The Models, Trotter's Formula and the Contours}

We shall consider several quantum lattice systems, for which we shall use both the magnetic and lattice gas language. (See Ref. [10] for the correspondence between the two of them.) The configuration space of the system is a $v$-dimensional cubic lattice $(v \geqq 2)$. With each site $r$ is associated a 2-dimensional vector space. Let $\sigma_{r}^{x, y, z}$ be the Pauli matrices acting on this space. In the lattice gas language, $\sigma_{r}^{ \pm}=\frac{1}{2}\left(\sigma_{r}^{x} \pm i \sigma_{r}^{y}\right)$ are the creation and annihilation operators for a particle on site $r$, and the number of particles is $n_{r}=\sigma_{r}^{+} \sigma_{r}^{-}=\left(1+\sigma_{r}^{z}\right) / 2$. A convenient basis 
in this space consists of the eigenvectors of $n_{r}$ (resp. $\sigma_{r}^{2}$ ), corresponding to the eigenvalues 1 and 0 (resp. 1 and -1 ) and describing occupation or emptiness (resp. spin up or down). The system is enclosed in a cubic box $\Lambda$ of volume $V$. The hamiltonian $H$ is the sum of two terms:

a longitudinal term $U$ (potential energy term), taken over from the corresponding classical problem. At this stage, the only relevant property of $U$ is that it is diagonal in the occupation number representation.

a transverse term $K$ (kinetic energy term).

We refer to the various models by the numbers given in the introduction. $K$ is then chosen as follows:

For the models (1), (2) and (5):

$$
K=-a \sum_{r \neq s}^{\prime} \sigma_{r}^{+} \sigma_{s}^{-}-b \sum_{r \neq s}^{\prime \prime} \sigma_{r}^{+} \sigma_{s}^{-}
$$

where $\sum^{\prime}$ means that the sum is restricted to the nearest neighbours, and $\sum^{\prime \prime}$ that it is restricted to the next-to-nearest-neighbours. One should be careful that, if one defines the distance between two lattice points by:

$$
|r-s|=\sum_{\alpha=1}^{v}\left|r_{\alpha}-s_{\alpha}\right|
$$

then $\sum^{\prime \prime}$ refers to pairs of points at a distance $|r-s|=2$ (and not $v$, if $v>2$ ). This choice will emerge as the most natural one in the following section.

Under the unitary transformation $\prod_{\frac{1}{2} r} \sigma_{r}^{z}$, where the product runs over every other site of the lattice, $U$ is invariant, $a$ changes sign and $b$ is invariant. It is therefore no restriction to take $a \geqq 0$. We also impose $b \geqq 0$, which, however, is a restriction.

For the models (3) and (4):

$$
K=-\frac{h}{2} \sum_{r} \sigma_{r}^{x}=-\frac{h}{2} \sum_{r}\left(\sigma_{r}^{+}+\sigma_{r}^{-}\right) .
$$

(3.3) describes a magnetic field in the positive $x$ direction $(h>0)$.

In order to apply the general theory of Section 2, we have to choose the observable $A_{A}$ and the projectors $P_{A}$ and $Q_{A}$. This will be done in close analogy with the classical case. Let $S_{A} \subset A$ be the set of sites on the boundary of the cube $\Lambda . P_{A}$ and $Q_{A}$ will be the projections on common eigensubspaces of all the $n_{r}$ (or $\sigma_{r}^{z}$ ) for all $r \in S_{A}$, with suitable eigenvalues. They will be defined therefore by these eigenvalues. We make the following choice:

For the models (2) and (4a) (ferromagnets):

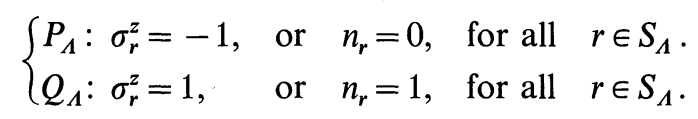


$A_{\Lambda}$ is the magnetization (one could equivalently take the density):

$$
A_{\Lambda}=V^{-1} \sum_{r \in \Lambda} \sigma_{r}^{z} \text {. }
$$

In these cases, the unitary transformation $W_{\Lambda}=\prod_{r \in \Lambda} \sigma_{r}^{x}$ exchanges $P_{\Lambda}$ and $Q_{\Lambda}$, and changes $A_{\Lambda}$ into $-A_{\Lambda}$. Furthermore, the longitudinal part $U$ of $H$ will be chosen to commute with $W_{A}$. Therefore, it will be sufficient to check that for some fixed $\eta>0$ :

$$
m_{A} \equiv\left\langle A_{A}\right\rangle_{P_{A}} \leqq-\eta<0 .
$$

For the models (1), (3), (4b) and (5) (antiferromagnets):

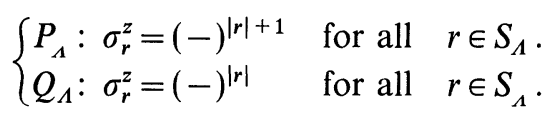

$A_{A}$ is the sublattice magnetization:

$$
A_{\Lambda}=V^{-1} \sum_{r \in \Lambda}(-)^{|r|} \sigma_{r}^{z}
$$

where $|r|$ is defined in analogy with (3.2).

Strictly speaking, no $W_{A}$ exists in this case, since the obvious candidates, namely the translations by one step parallel to one of the coordinate axes, do not leave $\Lambda$ invariant. This will not affect the conclusion, however, and it will still be sufficient to check (3.6).

All the $P_{A}, Q_{A}, A_{A}$ are diagonal in the occupation number representation, and (2.11) is obviously satisfied. We now concentrate on $m_{A}=\left\langle A_{A}\right\rangle_{P_{A}}$, and drop from now on the index $\Lambda$, as well as the projector $P_{\Lambda}$. Then:

where

$$
m \equiv\langle A\rangle=Z^{-1} \operatorname{Tr}(A \exp (-H))
$$

$$
Z=\operatorname{Tr} \exp (-H)
$$

and where the inverse temperatur $\beta$ is supposed to be included in the coefficients of $H$.

We apply Trotter's formula [8] to $\exp (-H)$ :

$$
\begin{aligned}
\exp (-H) & =\lim _{T \rightarrow \infty}\left(\exp \left(-\frac{U}{T}\right) \exp \left(-\frac{K}{T}\right)\right)^{T} \\
& =\lim _{T \rightarrow \infty}\left(\exp \left(-\frac{U}{T}\right)\left(1-\frac{K}{T}\right)\right)^{T}
\end{aligned}
$$

where $T$ is a positive integer. Since we are in fact dealing with finite dimensional matrices, the convergence problems are trivial. We substitute (3.12) into the definition of $m$ and expand the product by introducing intermediate states running over a basis of the range of $P$ between 
successive factors. We take this basis to consist of eigenvectors of all the $n_{r}(r \in \Lambda)$. Such a vector $|D\rangle$ corresponds in a one-to-one way to a classical configuration $D$ in $A . U$ and $A$ are diagonal in this representation. Let $U(D)=\langle D|U| D\rangle$ and similarly $A(D)=\langle D|A| D\rangle$. Then

$$
\begin{aligned}
\operatorname{Tr}[A \exp (-H)]= & \lim _{T \rightarrow \infty} \sum_{\{D(t)\}} A(D(1)) \exp \left[-\frac{1}{T} \sum_{t=1}^{T} U(D(t))\right] \\
& \cdot \prod_{t=1}^{T}\left\langle D(t)\left|1-\frac{K}{T}\right| D(t+1)\right\rangle
\end{aligned}
$$

Here $t$ is an integer, $1 \leqq t \leqq T$. $t$ will be called the time. $D(t)$ is the classical configuration that occurs at time $t$, which means that we have inserted $|D(t)\rangle\langle D(t)|$ between the $(t-1)$-st and the $t$-th factor in the product (3.12). We take of course $D(T+1)=D(1)$. The matrix elements in the last factor are non zero only in a small number of cases, depending on the choice of $K$. We consider separately the cases where $K$ is a transverse interaction or a transverse magnetic field.

For the models (1), (2) and (5), $K$ is the transverse interaction (3.1). The matrix element in (3.13) is non zero in the following cases:

(1) $D(t)$ and $D(t+1)$ are identical, in which case it is one.

(2) (resp. (3)) $D(t)$ and $D(t+1)$ differ by one particle and a neighbouring (resp. next-to-neighbouring) hole having exchanged their positions, in which case it is $a / T$ (resp. $b / T$ ). The latter case we describe as an $a$-jump (resp. $b$-jump).

Cases (2) and (3) we describe as jumps. A configuration of the system is now defined as a sequence $\Delta=\{D(t), t=1, \ldots, T+1\}$ of classical configurations in the box $\Lambda$, such that $D(T+1)=D(1)$, and for all $t$, $D(t)$ and $D(t+1)$ are related as above. The configurations of the quantum system therefore build a subset of the set of classical configurations in a box $\Omega=\Lambda \times[1, T+1]$ on a $(v+1)$-dimensional lattice. Let $\Delta$ be a configuration. Let $X_{a}(\Delta)$ and $X_{b}(\Delta)$ be respectively the number of $a$-jumps and $b$-jumps, and let:

Then

$$
U(\Delta)=\frac{1}{T} \sum_{t=1}^{T} U(D(t))
$$

$$
m=\lim _{T \rightarrow \infty} \frac{\sum_{\Delta} A(D(1)) \exp [-U(\Delta)]\left(\frac{a}{T}\right)^{X_{a}(\Delta)}\left(\frac{b}{T}\right)^{X_{b}(\Delta)}}{\sum_{\Delta} \exp [-U(\Delta)]\left(\frac{a}{T}\right)^{X_{a}(\Delta)}\left(\frac{b}{T}\right)^{X_{b}(\Delta)}}
$$

The effect of the projector $P_{A}$, which is not written explicitly, is to restrict the sum over $\Delta$ to those $\Delta$ for which for all $t, D(t)$ satisfies the boundary condition which defines $P_{\Lambda}$. 
Since $a>0, b>0$, all terms in (3.15) are positive. Therefore the quantum Gibbs distribution defines for each $T$ a probability measure over a set of configurations on a $(v+1)$-dimensional lattice. The probability of a configuration is:

$$
P(\Delta)=\exp [-U(\Delta)]\left(\frac{a}{T}\right)^{X_{a}(\Delta)}\left(\frac{b}{T}\right)^{X_{b}(\Delta)} / \sum_{\Delta} \text { idem. }
$$

The problem is now similar to the classical one.

For the models (3) and (4) $K$ is a transverse field (3.3). The analysis is almost identical. The matrix element in (3.13) is non zero if

(1) $D(t)$ and $D(t+1)$ are identical, in which case it is one,

(2) $D(t)$ and $D(t+1)$ differ by one site being occupied in one of them and empty in the other, in which case it is $h / 2 T$. This we still call a jump. Configurations $\Delta$ are defined accordingly. Let $X(\Delta)$ be the number of jumps of $\Delta$. Then:

$$
m=\lim _{T \rightarrow \infty} \frac{\sum_{\Delta} A(D(1)) \exp [-U(\Delta)]\left(\frac{h}{2 T}\right)^{X(\Delta)}}{\sum_{\Delta} \exp [-U(\Delta)]\left(\frac{h}{2 T}\right)^{X(\Delta)}}
$$

The probabilistic interpretation is similar to the previous one, with:

$$
P(\Delta)=\exp [-U(\Delta)]\left(\frac{h}{2 T}\right)^{X(\Delta)} / \sum_{\Delta} \text { idem . }
$$

We now describe some geometrical properties of configurations. The following considerations apply not only to the previous periodic configurations $(D(T+1)=D(1))$, but also to non-periodic ones, which would be needed for the study of operators that are not diagonal in the occupation number representation.

To each configuration, we first associate contours similar to the classical ones. Each point in $\Omega=\Lambda \times[1, T+1]$ is the center of a $(v+1)$ dimensional unit cube, hereafter called cube. A cube is occupied (resp. empty) if its center $r$ is occupied (resp. empty), namely if $n_{r}=1$ (resp. $n_{r}=0$ ). The faces of the cubes are $v$-dimensional unit cubes, hereafter called faces. The faces of the faces are $(v-1)$-dimensional unit cubes, hereafter called edges. We shall consider exclusively the faces and edges parallel to the time axis, and disregard all others. The set of cubes (resp. faces and edges) occuring at a given time can and will be identified with the set of their spatial projections, namely their projections on the $v$-dimensional space orthogonal to the time axis. These projections are $v$-dimensional (resp. $(v-1)$ and $(v-2)$-dimensional) unit cubes, and will again be called cubes (resp. faces and edges). 
Let $\Delta$ be a configuration. We now define effective faces in $\Delta$.

Definition 1. A face is effective in $\Delta$ if it satisfies the following condition:

(1) For the models (2) and (4a) (ferromagnets): one of the cubes adjacent to this face is occupied in $\Delta$, and the other one is empty.

(2) For the models (1), (2), (4b) and (5) (antiferromagnets): the two cubes adjacent to this face are both empty or both occupied in $\Delta$.

Let $\Delta$ satisfy the boundary conditions that define $P_{A}$ or $Q_{A}$. Let $\mathscr{E}(\Delta)$ be the set of effective faces in $\Delta$. Then the configuration $\Delta$ is uniquely defined by $\mathscr{E}(\Delta)$. The effective faces at time $t$ form closed polyhedra, which characterize the classical configuration $D(t)$.

The following is an equivalence relation between faces in $\mathscr{E}(\Delta)$.

Definition 2. Two faces $f$ and $f^{\prime}$ in $\mathscr{E}(\Delta)$ are connected if there exists a sequence $\left(f=f_{0}, f_{1}, \ldots, f_{n}=f^{\prime}\right)$ of faces in $\mathscr{E}(\Delta)$ such that for any $j=1, \ldots, n$, the pair of faces $\left(f_{j-1}, f_{j}\right)$ satisfy one of the following two conditions:

(1) They occur at the same time and have a common edge.

(2) They occur at consecutive times $(t, t \pm 1)$, and their spatial projections have a common edge.

We now define contours.

Definition 3. We call contours of $\Delta$ the equivalence classes of $\mathscr{E}(\Delta)$ under this relation. If $G$ is a contour of $\Delta$, we write $G \subset \Delta$.

We next describe some properties of contours.

Let $G$ be a contour, $G(t)$ the set of faces of $G$ at time $t$. $G(t)$ is identified with its spatial projection, which is a family of closed polyhedra in $v$-dimensional space. $G(t)$ is not connected in the usual sense in general. A point in $\Lambda$ is said to be internal (resp. external) for $G(t)$ if it can be reached from outside by crossing $G(t)$ an odd (resp. even) number of times.

If a face belongs to $G(t)$ and is not affected by a jump between $D(t)$ and $D(t+1)$, then it also belongs to $G(t+1)$. Suppose now that $\Delta$ has a jump between $D(t)$ and $D(t+1)$. It can be described as follows:

If $K$ is given by (3.3) (transverse field), then there is one cube with the following two properties:

$(\alpha)$ its center is in opposite situation (external vs. internal) with respect to $D(t)$ and $D(t+1)$,

$(\beta)$ each of its faces is effective at time $t$ but not $(t+1)$, or at $(t+1)$ but not $t$.

If $K$ is given by (3.1) (transverse interaction), and the jump is a $b$-jump, then there are two cubes with the properties $(\alpha)$ and $(\beta)$, and they are next-to-nearest-neighbours, which means that they have a common edge but no common face. 
If $K$ is given by (3.1) and the jump is an $a$-jump, then there are two cubes with properties $(\alpha)$ and $(\beta)$, except that they now have a common face, which is effective in both $D(t)$ and $D(t+1)$ or neither.

In all cases, it follows from the definitions that if one of the effective faces of the jumping cube (or cubes) at time $t$ or $(t+1)$ belongs to $G$, then all effective faces of the jumping cube (or cubes) at time $t$ and $(t+1)$ belong to $G$. We then say that the jump itself belongs to $G$.

From this it follows that for a given $G$, there exists a unique configuration $\Delta_{G}$ such that $\mathscr{E}\left(\Delta_{G}\right)=G$. The number of jumps (resp. $a$-jumps or $b$-jumps) of $\Delta_{G}$ we call number of jumps (resp. $a$-jumps or $b$-jumps) of $G$ and we denote by $X(G)\left(\operatorname{resp} . X_{a}(G)\right.$ or $\left.X_{b}(G)\right)$. Then clearly, for all $\Delta$ :

$$
X_{(a, b)}(\Delta)=\sum_{G \subset \Delta} X_{(a, b)}(G) .
$$

It is now clear in what sense the choice (3.1) is natural. The convenient notion to be used when defining classical contours is connectedness by edge. In (3.1) we have allowed all the jumps that do not destroy this connectedness. It would not be substantially simpler to consider only $a$-jumps.

Our final aim is to prove (3.6). We now give a sufficient condition for (3.6) to hold, which relies heavily on contours.

Lemma 3. A sufficient condition for (3.6) to hold is that the probability $\pi(r)$ for $r \in \Lambda$ to lie inside at least one contour at time 1 satisfies:

$$
\pi(r) \leqq \frac{1-\eta}{2}<\frac{1}{2} .
$$

Proof. The definitions of $P_{A}$ and contours have been chosen in such a way that any $r \in \Lambda$ which can be reached from outside without crossing any contour contributes -1 to $V A_{\Lambda}$. Therefore

$$
\begin{aligned}
\left\langle A_{\Lambda}\right\rangle_{P_{A}} & \leqq V^{-1} \sum_{r \in A}[\pi(r)-(1-\pi(r))] \\
& \leqq \sup _{r \in A}(2 \pi(r)-1) .
\end{aligned}
$$

The lemma follows immediately. Notice that $\pi(r)=0$ for $r \in S_{A}$.

There remains to obtain bounds on $\pi(r)$. This will result from three estimates, as sketched in the introduction: a probability estimate for an arbitrary contour $G$, an entropy or shape estimate, and a position or density estimate. The last one is the number of possible positions of $G$ with a given shape, such that $r$ be reached from outside by crossing $G(1)$ at least once. If $g \equiv g(1)$ is the total area of $G(1)$, this is less than $(g / 2 v)^{v / v-1}$ by the classical estimate. The first two estimates are model-dependent. 
In the following sections, we shall obtain these estimates and deduce from them the property (3.20) of $\pi(r)$, which implies (3.6) and therefore the existence of a phase transition.

\section{Quantum System with Extended Hard Cores}

The hamiltonian $H=K+U$ is defined by (3.1) and by

where:

$$
U=-\mu \sum_{r} n_{r}+\frac{1}{2} \sum_{r \neq s} \varphi(r-s) n_{r} n_{s}
$$

$$
\begin{gathered}
\varphi(r)=+\infty \text { for }|r|=1 \\
\sum_{|r| \geqq 2}|r||\varphi(r)|<\infty .
\end{gathered}
$$

The appropriate $A_{A}, P_{A}$ and $Q_{A}$ for this model are defined by $(3.7,8)$. For technical reasons $[5,12]$, we restrict the length of the edge of $\Lambda$ to be even. The extended hard core considerably restricts the possible configurations and contours. Effective faces can only separate empty cubes.

Let $g(t)$ be the total area of $G(t)$, and $g_{\alpha}(t)$ the area of the faces of $G(t)$ that are orthogonal to the $\alpha$-th coordinate axis. Then, for all $\alpha=1, \ldots, v$, and all $t, g(t)=v g_{\alpha}(t)$. Therefore $g(t)=2 v k(t)$, where $k(t)$ is an integer. The only possible jumps are shown on Figs. 1 and 2 (respectively $a$-jump

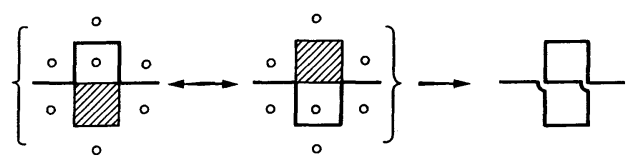

Fig. 1. Typical $a$-jump for model (1)

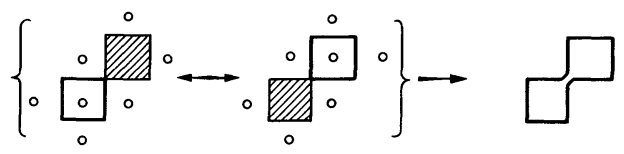

Fig. 2. Typical $b$-jump for model (1)

and $b$-jump), where shaded means occupied, a small circle means empty, and no claim is made concerning the effectiveness of the faces not drawn. In particular, one sees immediately that $g(t)$ is independent of $t$, so that $k(t)=k(1) \equiv k$. If $G$ is a contour, we denote by $X_{a}, X_{b}$ and $X=X_{a}+X_{b}$ the number of $a$-jumps, of $b$-jumps and of jumps of $G$. We now derive the relevant estimates. 
Lemma 4. The probability $P(G)$ of a given contour $G$ satisfies:

where:

$$
P(G) \leqq \exp [-(\mu-c) k]\left(\frac{a}{T}\right)^{X_{a}}\left(\frac{b}{T}\right)^{X_{b}}
$$

$$
c=\frac{1}{v} \sum_{\alpha} \sum_{r:|r|>1}\left(\left|r_{\alpha}\right|+1\right)|\varphi(r)| .
$$

Proof. Up to an inessential improvement in estimating the contribution of the tail of the potential, the proof is a straightforward transcription of that in [5] and will not be repeated. The transformation $\tilde{T}_{G}$ which occurs in [5] is replaced by

$$
\tilde{\mathscr{T}}_{G}=\prod_{t} \tilde{T}_{G(t)} .
$$

We need also an entropy estimate.

Lemma 5. The number of possible contour shapes with given $g=2 v k$ and given $X_{a}$ and $X_{b}$ is at most:

$$
\begin{aligned}
& S\left(k, X_{a}, X_{b}\right) \leqq \frac{T !}{X_{a} ! X_{b} !(T-X) !} 2^{(2 v+1) k-v} \\
& \cdot\left(2^{2 v+2} 2 v k\right)^{X}\left(\frac{2}{2 v-1}\right)^{X_{a}}(v-1)^{X_{b}} .
\end{aligned}
$$

Proof. We first estimate the number of possible shapes of $G(1)$, for given $g$ and $X$. The classical estimate $2^{g-v}$, which corresponds to $X=0$, no longer holds because $G(1)$ is not connected in general. Now, with each jump of $G$ is associated a pair of cubes. It follows immediately from Defs. 2 and 3 that by adding to $G(1)$ the $2 X$ jumping cubes, we obtain a connected polyhedron. Moreover, it is clear by inspection of Figs. 1 and 2, that as far as connectedness is concerned, we loose nothing by adding only one cube per jump, namely the jumping cube that is occupied before the jump. Adding these $X$ cubes to $G(1)$, we then obtain a connected polyhedron $\bar{G}$ with area $g+2 v X$. Now $\bar{G}$ can be considered as a single closed polyhedron, by which we mean that the faces of $\bar{G}$ adjacent to the same edge are connected in pairs, in such a way that the set of all faces of $\bar{G}$ is pairwise connected, and that no face has a free edge. In fact, $\bar{G}$ can be constructed by adding $X$ times a cube to some polyhedron, in the situation shown on Figs. 1 and 2. One then opens the polyhedron and the cube along a common edge, and reconnects the faces as shown on the same figures. Furthermore, this can be done in such a way that no two directly connected faces of $\bar{G}$ are parallel. The number of possible shapes of $\bar{G}$ is therefore at most $2^{g+2 v X-v}$ by the classical estimate $[2,5,7]$, where however 3 is replaced by 2 in view of the restriction mentioned above. 
In order to extract $G(1)$ from $\bar{G}$, it is sufficient to extract $X$ cubes. Each one is characterized by its lower horizontal face: one has therefore to choose $X$ faces among $g / v+2 X$. Since each choice removes one cube and therefore two horizontal faces, one finally gets:

$$
2^{X}\left(\begin{array}{c}
k+X \\
X
\end{array}\right) \leqq 2^{k+2 X}
$$

$G(1)$ being chosen, one then chooses the times at which the jumps occur. The number of possible choices is:

$$
\frac{T !}{X_{a} ! X_{b} !(T-X) !} .
$$

We now complete the counting of the possible $G$ by estimating the number of possible $G(t)$ in the order of increasing $t$. Suppose $G(t)$ is fixed. If there is no jump at time $t$, then $G(t+1)=G(t)$. If there is an $a$-jump at time $t$, the number of possible $a$-jumps, and therefore of possible $G(t+1)$, is at most equal to the number of non effective faces of cubes, the $(2 v-1)$ other faces of which are effective in $G(t)$ (see Fig. 1). Since each face of $G(t)$ can be used at most twice as a face of such an incomplete cube, this number is less that $\frac{2 g}{2 v-1}$. This is best possible, as shown by a close-packing arrangement of crosses of the type represented by Fig. 3. If there is a $b$-jump at time $t$, the number of possible $b$-jumps

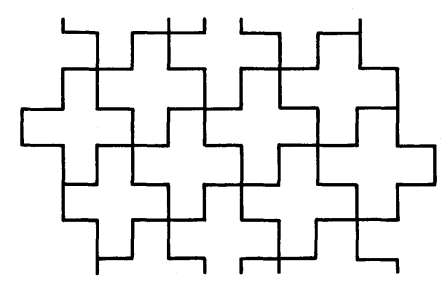

Fig. 3. Contour with the maximum number of possible $a$-jumps for model (1)

is at most equal to the number of edges of $G(t)$, namely $(v-1) g$. Collecting all the estimates, we obtain (4.7).

We have all the estimates needed. In order to formulate the result, we reintroduce the inverse temperature $\beta$. Let:

$$
\mu=\beta \tilde{\mu}, \quad a=\beta \tilde{a}, \quad b=\beta \tilde{b}, \quad \varphi=\beta \tilde{\varphi}, \quad \text { etc. }
$$


Theorem 1. The model defined by (3.1) and (4.1) exhibits a phase transition for all $\tilde{\mu}>\tilde{\mu}_{0}$ and $\beta>\beta_{0}(\tilde{\mu})$, where

$$
\tilde{\mu}_{0}=\tilde{c}+2^{2 v+3}\left(\frac{2 v}{2 v-1} \tilde{a}+v(v-1) \tilde{b}\right) .
$$

The function $\beta_{0}(\tilde{\mu})$ is a decreasing function of $\tilde{\mu}$. It tends to infinity when $\tilde{\mu}$ tends to $\tilde{\mu}_{0}$, and tends to zero when $\tilde{\mu}$ tends to infinity.

The two-phase region is indicated by shading on Fig. 4 , in the $(\beta-\tilde{\mu})$ plane, for fixed $\tilde{a}, \tilde{b}$ and $\tilde{\varphi}$.

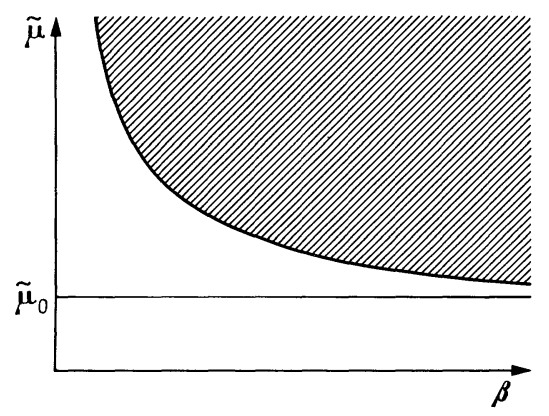

Fig. 4. Two-phase region for model (1) for fixed $(\tilde{a}, \tilde{b}, \tilde{\varphi})$

Proof. It is sufficient to prove (3.20). We collect the estimates from Lemmas 4 and 5, and obtain:

$$
\begin{aligned}
\pi(r) \leqq & 2^{-v} \sum_{k \geqq 1} k^{\frac{v}{v-1}} 2^{(2 v+1) k} e^{-(\mu-c) k} \sum_{X_{a}, X_{b}}\left(\frac{a}{T}\right)^{X_{a}} \\
& \cdot\left(\frac{b}{T}\right)^{X_{b}} \frac{T !}{X_{a} ! X_{b} !(T-X) !}\left(2^{2 v+3} v k\right)^{X}\left(\frac{2}{2 v-1}\right)^{X_{a}}(v-1)^{X_{b}} .
\end{aligned}
$$

The last sum is equal to:

$$
\begin{aligned}
\sum_{X}\left(\begin{array}{c}
T \\
X
\end{array}\right)\left(\frac{2^{2 v+3} v k}{T}\right)^{X} & \left(\frac{2 a}{2 v-1}+(v-1) b\right)^{X} \\
= & {\left[1+\frac{1}{T} 2^{2 v+3}\left(\frac{2 v a}{2 v-1}+v(v-1) b\right) k\right]^{T} . }
\end{aligned}
$$

Therefore:

$$
\begin{aligned}
\pi(r) \leqq 2^{-v} & \sum_{k \geqq 1} k^{\frac{v}{v-1}} 2^{(2 v+1) k} \\
& \cdot \exp \left[-k\left(\mu-c-2^{2 v+3}\left(\frac{2 v a}{2 v-1}+v(v-1) b\right)\right)\right] .
\end{aligned}
$$


The series can be made convergent and the sum arbitrarily small, and in particular less than $1 / 2$, by taking $\mu$ sufficiently large. Note also that the last exponent is linear in $\beta$. In particular, for all $\tilde{\mu}, \tilde{a}, \tilde{b}$ and $\tilde{\varphi}$ that satisfy $\tilde{\mu}>\tilde{\mu}_{0}$, a phase transition will occur for $\beta$ sufficiently large. This proves Theorem 1.

Remark 1. Instead of proceeding as above, one could also deduce a bound on the entropy from the fact that, when $G(1)$ and the $X$ cubes are known, as well as the jumping times, then $G$ is uniquely determined by the order in which the jumping cubes are used, which introduces a factor $X$ !. The last sum in (4.12) would then be replaced by:

$$
\sum_{X}\left(\begin{array}{c}
T \\
X
\end{array}\right) X ! 2^{(2 v+2) X}\left(\frac{a}{T}\right)^{X_{a}}\left(\frac{b}{T}\right)^{X_{b}} .
$$

The fact that a jump is $a$ or $b$ is now determined uniquely by the geometrical situation. We therefore obtain:

$$
\begin{aligned}
\ldots & \leqq \sum_{X} \frac{T !}{T^{X}(T-X) !}\left(2^{2 v+2} \operatorname{Max}(a, b)\right)^{X} \\
& \leqq\left[1-2^{2(v+1)} \operatorname{Max}(a, b)\right]^{-1} .
\end{aligned}
$$

However, this is restricted to $\operatorname{Max}(a, b)<2^{-2(v+1)}$. In particular, the allowed values $\tilde{a}$ and $\tilde{b}$ of the transverse interaction would tend to zero like $\beta^{-1}$ for $\beta \rightarrow \infty$. This estimate is therefore of restricted interest.

\section{Anisotropic Heisenberg Ferromagnet}

The hamiltonian $H=K+U$ is defined by (3.1) and by:

$$
\begin{aligned}
U & =\frac{1}{4} \sum_{r<s} \varphi(r-s)\left(\sigma_{r}^{z} \sigma_{s}^{z}-1\right) \\
& =-\frac{1}{2} \sum_{r<s} \varphi(r-s)\left[n_{r}\left(1-n_{s}\right)+n_{s}\left(1-n_{r}\right)\right]
\end{aligned}
$$

where:

and:

$$
\varphi(r)=-2 a^{\prime}<0 \text { for }|r|=1
$$

$$
\operatorname{Max}_{\alpha} \sum_{r:|r|>1}\left|r_{\alpha}\right||\varphi(r)|<4 v a^{\prime} .
$$

The appropriate $A_{\Lambda}, P_{A}$ and $Q_{\Lambda}$ for this model are defined by $(3.4,5)$. The effective faces separate occupied cubes from empty cubes. Therefore all jumps are superficial, in the sense that they exchange two cubes, one of which is internal and the other external to the contour $G$ to which the jumps belong. For an $a$-jump from $G(t)$ to $G(t+1)$, the jumping cubes have a common face, referred to as the jumping face, which belongs to both $G(t)$ and $G(t+1)$. The jumping face uniquely determines the

16 Commun. math. Phys., Vol. 14 
jump. For a $b$-jump, the jumping cubes have no common face but only a common edge, which also belongs to both $G(t)$ and $G(t+1)$. A given edge of $G(t)$ or $G(t+1)$ can accommodate at most two jumps. In order to describe the times at which the jumps of a given $G$ occur, it will be convenient to introduce the following notation: We define a function $x(t)(1 \leqq t \leqq T)$ by:

$$
\begin{cases}x(t)=0 & \text { if there is no jump from } G(t) \text { to } G(t+1) \\ x(t)=1 & \text { if there is a jump from } G(t) \text { to } G(t+1)\end{cases}
$$

Let:

$$
X(t)=\sum_{s=1}^{t} x(s)
$$

Then, the total number of jumps of $G$ is $X=X(T)$. We still denote by $X_{a}$ and $X_{b}$ the numbers of $a$-jumps and $b$-jumps $\left(X=X_{a}+X_{b}\right)$.

In this model, $g(t)$ is not constant in general. However, one sees immediately by inspection that:

$$
|g(t+1)-g(t)| \leqq(4 v-2) x(t) .
$$

From this it follows that:

$$
|g(t+1)-g| \leqq(4 v-2) X(t) .
$$

One could in fact consider separately $a$-jumps and $b$-jumps. For the latter, the coefficient $(4 v-2)$ can be replaced by $(4 v-4)$. We shall not make this negligible improvement.

We now derive the relevant estimates.

Lemma 6. The probability of a given contour satisfies:

where

$$
P(G) \leqq \exp \left[-\frac{c}{T} \sum_{t=1}^{T} g(t)\right]\left(\frac{a}{T}\right)^{X_{a}}\left(\frac{b}{T}\right)^{X_{b}}
$$

$$
c=a^{\prime}-\frac{1}{4 v} \operatorname{Max}_{\alpha} \sum_{r:|r|>1}\left|r_{\alpha}\right||\varphi(r)| .
$$

Proof. For nearest neighbour interaction, the proof is a straightforward transcription of the classical one [2 to 7] and will be omitted. The inclusion of the tail of the potential follows the argument in Ref. [7], up to a trivial improvement by a factor 2 .

We need also an entropy estimate.

Lemma 7. The number of possible contour shapes with given $g \equiv g(1)$, given $x($.$) and X_{a}$ (therefore also given $X$ and $X_{b}$ ) is at most:

$S\left(g, x(),. X_{b}\right) \leqq\left(\begin{array}{l}X \\ X_{b}\end{array}\right) 3^{g+2 v X-v} \cdot 2^{g / 2 v+2 X}[2(v-1)]^{X_{b}} \prod_{t=1}^{T}(g(t))^{x(t)}$. 
Before proving Lemma 7, we make the following remark. It may seem surprising that (5.11) still depends on $g($.$) ; the reason is the following:$ we estimate the number of contour shapes in successive steps (in fact successive values of $t$ ), and the number of possibilities at each step depends on the choice that has been made at the previous steps, namely the choice of the preceding $G(t)$. The estimate (5.11) will be used in conjunction with (5.9), which also depends on the same choice, and we shall take the supremum over all possible $g(t)$ compatible with (5.7) only after combining (5.9) and (5.11). Taking the supremum over $g(t)$ separately in (5.9) and (5.11) before combining them would lead to a catastrophic result.

Proof of Lemma 7. The proof is very similar to that of Lemma 5. We first estimate the number of possible shapes of $G(1)$ for given $g$ and $X$. With each jump of $G$ is associated in a one-to-one way a pair of cubes. It follows from Defs. 2 and 3 that by adding to $G(1)$ the $2 X$ jumping cubes, we obtain a connected polyhedron. We can improve on this result by using the periodicity condition $G(T+1)=G(1)$ : it implies that after $X$ jumps, each site is in the same occupation state as it was initially. Now this state changes whenever a jumping cube is added around the site in question. Therefore, all the jumping cubes occur at least twice. It is therefore sufficient to add $X$ cubes to $G(1)$ to obtain a connected polyhedron $\bar{G}$, the area of which is then $g+2 v X$. In $\bar{G}$, each face occurs as many times as it does in $G(1)$ and the jumping cubes. In particular, all jumping faces associated with $a$-jumps occur at least twice in $\bar{G}$.

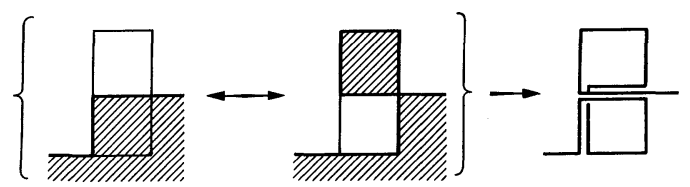

Fig. 5. Typical $a$-jump for model (2)

Now $\bar{G}$ can be considered as a single closed connected polyhedron, as one sees by opening the $X$ jumping cubes along suitable edges and sticking them along these edges to $G(1)$ or between themselves. This can be done without connecting two coinciding faces directly. A typical situation is shown in Fig. 5. The number of possible $\bar{G}$ is then at most $3^{g+2 v X-v}$ by the classical estimate. The number of possible $G(1)$ that can be extracted from $\bar{G}$ is at most $2^{g / 2 v+2 X}$, by the same reasoning as in the previous case (cf. (4.8)).

We have fixed $x($.$) and X_{b}$. We know therefore the jumping times and the number of jumps of each type. There are then $\left(\begin{array}{l}X \\ X_{b}\end{array}\right)$ possible choices
for the times and types of all jumps. 
This being fixed as well as $G(1)$, we then estimate the number of possible choices for $G(t)$ in the order of increasing $t$. For a given $G(t)$, there are $g(t)$ possible jumping faces for an $a$-jump, and $(v-1) g(t)$ edges, each of which can be used in two different ways, for a $b$-jump. The number of possible $G(t+1)$ if $x(t)=1$ is therefore $g(t)$ for an $a$-jump, and $2(v-1) g(t)$ for a $b$-jump. Collecting all the estimates, we obtain (5.11).

We have all the estimates needed. We reintroduce the temperature by (4.10) to formulate the result.

Theorem 2. The anisotropic Heisenberg ferromagnet defined by (3.1) and (5.1) exhibits a first order phase transition if the ratio of transverse to longitudinal interaction is sufficiently small and the temperature sufficiently low.

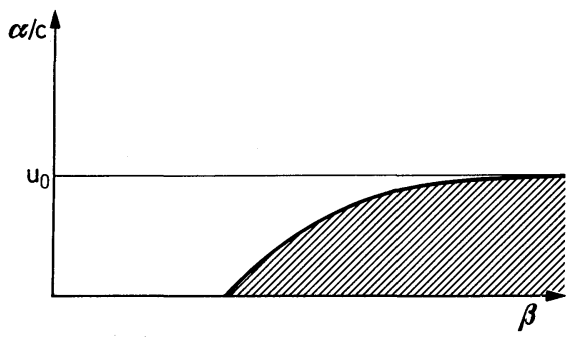

Fig. 6. Two-phase region for model (2)

The region where the existence of the phase transition is proved to exist is indicated on Fig. 6 in the plane of the variables $\beta$ and $\alpha / c$, for fixed $\tilde{\varphi}$, where $c$ is given by (5.10), and $\alpha$ by:

$$
\alpha=4.3^{2 v}(a+2(v-1) b)
$$

$\alpha / c$ is essentially the anisotropy. For nearest neighbour interactions, $\alpha / c=4.3^{2 v} \frac{a}{a^{\prime}}$. The isotropic Heisenberg model corresponds to $a=a^{\prime}$.

Proof of Theorem 2. It is again sufficient to prove (3.20). Collecting the estimates (5.9) and (5.11), we obtain:

$$
\begin{aligned}
& \pi(r) \leqq 3^{-v} \sum_{g \geqq 2 v}\left(\frac{g}{2 v}\right)^{\frac{v}{v-1}} 3^{g} 2^{g / 2 v} \sum_{x(.)}\left(43^{2 v}\right)^{X} \operatorname{Sup}_{g(.)} \\
& \cdot \exp \left[-\frac{c}{T} \sum_{t=1}^{T} g(t)\right] \prod_{t=1}^{T}(g(t))^{x(t)} \sum_{X_{b}}\left(\begin{array}{l}
X \\
X_{b}
\end{array}\right)\left(\frac{a}{T}\right)^{X_{a}}\left(2(v-1) \frac{b}{T}\right)^{X_{b}}
\end{aligned}
$$

where the supremum has to be taken over all $g(t)(t \geqq 2)$ compatible with (5.7) for fixed $g \equiv g(1)$. 
The last sum reduces to:

$$
\left[\frac{1}{T}(a+2(v-1) b)\right]^{X} \text {. }
$$

Substituting (5.14) into (5.13), we obtain for the second sum the following expression:

$$
S(g)=\sum_{x(.)} \operatorname{Sup}_{g(.)} \exp \left[-\frac{c}{T} \sum_{t=1}^{T} g(t)\right] \prod_{t=1}^{T}\left(\frac{\alpha}{T} g(t)\right)^{x(t)}
$$

where $\alpha$ is given by (5.12). We perform the sum over $x(t)$ in the order of decreasing $t$. This will require to perform $T$ times a sum of the type

$$
\sum_{x(t)}\left[\frac{\alpha}{T} g(t)\right]^{x(t)} \exp \left[-\frac{A(T-t)}{T} g(t+1)\right] .
$$

The sum starts with the term $t=T$, with $A(0)=0$. Taking at the same time the supremum over $g(t+1)$ for fixed $g(t)$ according to (5.7), we obtain:

$$
\begin{aligned}
\exp [ & \left.-\frac{A(T-t)}{T} g(t)\right]\left\{1+\frac{\alpha}{T} g(t) \exp \left[(4 v-2) \frac{A(T-t)}{T}\right]\right\} \\
& \leqq \exp \left[\left(-\frac{A(T-t)}{T}+\frac{\alpha}{\mathrm{T}} \exp \left[(4 v-2) \frac{A(T-t)}{T}\right]\right) \mathrm{g}(\mathrm{t})\right]
\end{aligned}
$$

Therefore the sum in (5.15) reduces to $T$ sums of the type (5.16), where $A(t)$ is determined by $A(0)=0$ and by the recurrence relation:

$$
A(t+1)=A(t)+c-\alpha \exp \left[(4 v-2) \frac{A(t)}{T}\right] .
$$

One then obtains

$$
S(g) \leqq \exp \left[-\frac{A(T)}{T} g\right]
$$

It is convenient to perform the harmless limit $T \rightarrow \infty$ at this stage. Let $\tau=t / T$ and

$$
u(\tau)=\exp \left[-(4 v-2) \frac{A(t)}{T}\right]
$$

Then $u(0)=1$; we need $u(1)$, and (5.19) becomes:

$$
\frac{d u}{d \tau}=(4 v-2)(\alpha-c u) \text {. }
$$

This gives immediately:

$$
u \equiv u(1)=\frac{\alpha}{c}+\left(1-\frac{\alpha}{c}\right) \exp [-(4 v-2) c] .
$$


Finally we obtain:

$$
\pi(r) \leqq 3^{-v} \sum_{g \geqq 2 v}\left(\frac{g}{2 v}\right)^{\frac{v}{v-1}} 3^{g} 2^{g / 2 v} u^{g /(4 v-2)} .
$$

This can be made arbitrarily small and in particular less than $1 / 2$, for $u$ sufficiently small. The condition for a phase transition is of the type $u<u_{0}$, and gives rise to the two phase region indicated on Fig. 6. This completes the proof of Theorem 2.

Remark 2. Instead of proceeding as above, one could also construct an entropy estimate containing $X$ !, where $X$ is the number of jumps of $G$. Used in conjunction with (5.8) and (5.9) this would also give a region of existence of two phases, but the allowed anisotropy would then tend to zero exponentially for $\beta$ tending to infinity, instead of increasing to a constant value.

\section{System with Extended Hard Cores and Transverse Field}

The hamiltonian is $H=K+U$ where $K$ is given by (3.3) and $U$ by (4.1). The reason for considering this strange model is pedagogical. It is the simplest of a family of three models ((3), (4) and (5) in the introduction) for which a new difficulty appears: in the models (1) and (2), all the jumps were superficial, in the sense that the jumping cubes at time $t$ were connected with $G(t)$. This will no longer be the case, and jumps from $G(t)$ will be possible anywhere. Of course the jumping cubes will still be connected either with $G(t)$ or with $G(t+1)$, but the entropy estimates will be more complicated, and the results weaker. In particular, for $\beta$ tending to infinity, the allowed anisotropy will have to tend to zero like $\beta^{-\frac{1}{2}}$, instead of increasing to a constant.

The present model uses the same $A_{A}, P_{A}$ and $Q_{A}$ as model (1). For fixed $t$, its contours are the same as those of model (1). Let $G$ be a contour. There are two possible types of jumps transforming $G(t)$ into $G(t+1)$ :

(1) jumps that create a particle, or equivalently destroy a unit cube from $G(t)$. These we call $y$-jumps or superficial jumps.

(2) jumps that destroy a particle, or equivalently add a unit cube to $G(t)$. These we call $z$-jumps or internal jumps.

The two notions are of course interchanged if we consider the successive values of $t$ in the opposite order. For a given $G$, we define $y(t)$ and $z(t)$ by:

$$
\begin{aligned}
& \begin{cases}y(t)=1 & \text { if } G \text { has a } y \text {-jump from } G(t) \text { to } G(t+1) \\
y(t)=0 & \text { otherwise. }\end{cases} \\
& \begin{cases}z(t)=1 & \text { if } G \text { has a } z \text {-jump from } G(t) \text { to } G(t+1) \\
z(t)=0 & \text { otherwise. }\end{cases}
\end{aligned}
$$


Then, if $g(t)=2 v k(t)$ :

Let

$$
k(t+1)=k(t)-y(t)+z(t) .
$$

and

$$
\left\{\begin{aligned}
Y(t) & =\sum_{s \leqq t} y(s) \\
Y^{\prime}(t) & =\sum_{s \geqq t} y(s)
\end{aligned}\right.
$$

$$
\left\{\begin{array}{l}
Z(t)=\sum_{s \leqq t} z(s) \\
Z^{\prime}(t)=\sum_{s \geqq t} z(s) .
\end{array}\right.
$$

Then:

$$
k(t+1)=k-Y(t)+Z(t) .
$$

The number of $y$-jumps (resp. $z$-jumps) is $Y=Y(T)=Y^{\prime}(1)$ (resp. $\left.Z=Z(T)=Z^{\prime}(1)\right)$. The total number of jumps is $X=Y+Z$.

The periodicity condition $G(T+1)=G(1)$ implies that each jumping cube occurs the same number of times for $y$-jumps and $z$-jumps. In particular, $Y=Z=X / 2$.

We now obtain the relevant estimates.

Lemma 8. The probability $P(G)$ of a contour $G$ satisfies:

$$
P(G) \leqq \exp \left[-\frac{(\mu-c)}{T} \sum_{t=1}^{T} k(t)\right]\left(\frac{h}{2 T}\right)^{X}
$$

where $c$ is given by (4.5).

Proof. The proof is identical with that of Lemma 4.

We need also an entropy estimate:

Lemma 9. The number of possible contour shapes with given $k, y($. and $z($.$) is at most:$

$$
S(k, y(.), z(.)) \leqq 2^{(2 v+1) k-v+(v+1) X} \prod_{t=1}^{T}[2 k(t)]^{y(t)}\left(Z^{\prime}(t)\right)^{z(t)}
$$

where $k(t)$ is given by (6.4).

Proof. By the same argument as in the proof of Lemma 5, there are at most $2^{(2 v+1) k-v+(v+1) X}$ ways to choose $G(1)$, with $g(1)=2 v k$, and the $X / 2$ cubes that characterize the jumps; each of these cubes has to be used once for a $y$-jump and once for a $z$-jump.

We next estimate the number of possible $G(t)$ in the order of increasing $t$. Let $G(t)$ be fixed. If $G$ has a $y$-jump at $t$, the number of possible $G(t+1)$ is at most equal to the number of unit cubes in $G(t)$, which is less than $g(t) / v=2 k(t)$. If $G$ has a $z$-jump, its cube has to be chosen among those of the $X / 2$ jumping cubes which have not been used before for $z$-jumps. There are $Z^{\prime}(t)$ of them. The lemma follows immediately. 
We have all the estimates needed. We reintroduce the temperature by (4.10).

Theorem 3. The model defined by (3.3) and (4.1) exhibits a phase transition for all $\tilde{\mu}>\tilde{c}, \beta>\beta_{0}(\tilde{\mu})$ and $\tilde{h} \leqq \tilde{h}_{0}(\beta, \tilde{\mu}) . \beta_{0}(\tilde{\mu})$ is an estimate of the classical type [5]: $\beta_{0}(\tilde{\mu})=C t(\tilde{\mu}-\tilde{c})^{-1} \cdot h_{0}(\beta, \tilde{\mu})$ is a function of the form $h_{0}(\beta, \tilde{\mu})=\beta^{-1} h_{0}(\mu-c)$, where $h_{0}(x) \approx x^{\frac{1}{2}}$ for $x \rightarrow \infty$. In particular, for fixed $\tilde{\mu}>\tilde{c}, h_{0}(\beta, \tilde{\mu})$ tends to zero like $\beta^{-\frac{1}{2}}$ when $\beta$ tends to infinity.

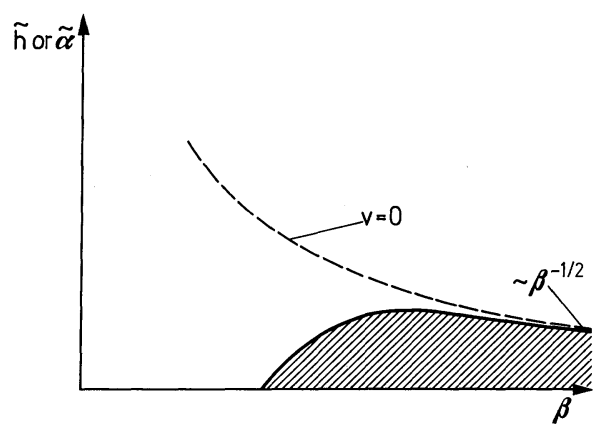

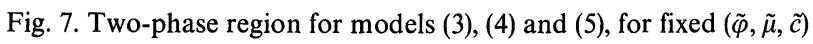

The shape of the two-phase region is shown on Fig. 7 for fixed $\tilde{\mu}, \tilde{\varphi}$, in the $(\beta, \tilde{h})$ plane

Proof. It is sufficient to prove (3.20). We collect the estimates from Lemmas 8 and 9 and obtain:

where

$$
\pi(r) \leqq 2^{-v} \sum_{k \geqq 1} k^{\frac{v}{v-1}} 2^{(2 v+1) k} S(k)
$$

$$
\begin{aligned}
S(k)=\sum_{y(.), z(.)} \exp & {\left[-\frac{\mu-c}{T} \sum_{t=1}^{T}(k-Y(t-1)+Z(t-1))\right] } \\
\cdot & \prod_{t=1}^{T}\left(\frac{\alpha Z^{\prime}(t)}{T}\right)^{z(t)}\left[\frac{2 \alpha}{T}(k-Y(t-1)+Z(t-1))\right]^{y(t)}
\end{aligned}
$$

where $\alpha=2^{v} h$. We obtain an upper bound for $S(k)$ by forgetting the restrictions $Z=Y$ and $y(t) . z(t)=0$. We first sum over $y($.$) for fixed$ $z($.$) and therefore fixed K(t)=k+Z(t)$. We consider:

$$
\sum_{y(.)} \exp \left[\frac{\mu-c}{T} \sum_{t=1}^{T} Y(t-1)\right] \prod_{t}\left[\frac{2 \alpha}{T}(K(t-1)-Y(t-1))\right]^{y(t)} .
$$


We compute this sum in the order of decreasing $t$ by performing $T$ times the following sum:

$$
\begin{aligned}
\sum_{y(t)} & \exp \left[\frac{A(T-t)}{T} Y(t)\right]\left[\frac{2 \alpha}{T}(K(t-1)-Y(t-1))\right]^{p(t)} \\
& \leqq \exp \left[\frac{A(T-t)}{T} Y(t-1)+\frac{2 \alpha}{T}(K(t-1)-Y(t-1)) \exp \left(\frac{A(T-t)}{T}\right)\right]
\end{aligned}
$$

If we determine $A(t)$ by $A(0)=0$ and the recurrence relation:

$$
A(t+1)=A(t)+(\mu-c)-2 \alpha \exp \left(\frac{A(t)}{T}\right)
$$

we obtain, after the summation over $y($.$) :$

$S(k) \leqq \exp \left(-k \frac{A(T)}{T}\right) \sum_{z(.)} \exp \left[-\frac{1}{T} \sum_{t=1}^{T} z(t) A(T-t)\right] \prod_{t}\left[\frac{\alpha}{T} Z^{\prime}(t)\right]^{z(t)}$.

We now perform the summation over $z($.$) in the order of increasing t$, by performing $T$ times a sum of the following type:

$$
\begin{aligned}
\sum_{z(t)} \exp \left[\frac{B(t-1)}{T} Z^{\prime}(t)\right]\left[\frac{\alpha}{T} Z^{\prime}(t) \exp \left(-\frac{A(T-t)}{T}\right)\right]^{z(t)} \\
\quad \leqq \exp \left[\frac{B(t-1)}{T} Z^{\prime}(t+1)+\frac{\alpha}{T}\left(Z^{\prime}(t+1)+1\right) \exp \left(\frac{B(t-1)-A(T-t)}{T}\right)\right] .
\end{aligned}
$$

If we determine $B(t)$ by $B(0)=0$ and the recurrence relation:

$$
B(t+1)=B(t)+\alpha \exp \left(\frac{B(t)-A(T-t-1)}{T}\right)
$$

we obtain, after the summation over $z($.$) :$

$$
S(k) \leqq \exp \left[-k \frac{A(T)}{T}+\frac{B(T)}{T}\right] .
$$

There remains only to compute $A(T) / T$ and $B(T) / T$. We perform the limit $T \rightarrow \infty$ at this stage. Let $\tau=t / T$, and let $u(\tau)=\exp (-A(T) / T)$, $v(\tau)=\exp [-B(T) / T]$. Then $u(0)=v(0)=1$. We need $u(1)$ and $v(1)$. The recurrence (6.11) is very similar to (5.19). One obtains:

$$
u(\tau)=\frac{2 \alpha}{\mu-c}+\left(1-\frac{2 \alpha}{\mu-c}\right) \exp [-(\mu-c) \tau] .
$$


The recurrence relation (6.14) becomes:

Therefore

$$
\frac{d v(\tau)}{d \tau}=-\alpha u(1-\tau)
$$

$v(\tau)=1-\frac{2 \alpha^{2}}{\mu-c} \tau-\frac{\alpha}{\mu-c}\left(1-\frac{2 \alpha}{\mu-c}\right)\left[e^{-(\mu-c)(1-\tau)}-e^{-(\mu-c)}\right]$.

Collecting all the estimates, we obtain:

where:

$$
\pi(r) \leqq 2^{-v} \sum_{k \geqq 1} k^{\frac{v}{v-1}} 2^{(2 v+1) k} u^{k} v^{-1}
$$

and

$$
u \equiv u(1)=\frac{2 \alpha}{\mu-c}+\left(1-\frac{2 \alpha}{\mu-c}\right) \exp [-(\mu-c)]
$$

$v \equiv v(1)=1-\frac{2 \alpha^{2}}{\mu-c}-\frac{\alpha}{\mu-c}\left(1-\frac{2 \alpha}{\mu-c}\right)(1-\exp [-(\mu-c)])$.

The estimate (6.19) however, holds only if $v$ determined by (6.21) is positive. The theorem follows immediately from $(6.19,20,21)$. In particular, for $\beta \rightarrow \infty$, the condition $v>0$ becomes $2 \alpha^{2}<(\mu-c)$, and therefore $\tilde{h}<C t \beta^{-\frac{1}{2}}$, for fixed $\tilde{\mu}$ and $\tilde{\varphi}$.

Remark 3. In this case also, we can derive a simpler entropy estimate containing $(Y+Z)$ ! The allowed magnetic field then decreases exponentially with $\beta$ when $\beta$ tends to infinity.

\section{Ising Model with Transverse Field}

The treatment in this section covers two different models with the same $K$ given by (3.3), and $U$ given either by $(5.1,2,3,4)$ for the Ising ferromagnet, or by:

where:

$$
U=\frac{1}{4} \sum_{r<s} \varphi(r-s)\left(\sigma_{r}^{z} \sigma_{s}^{z}+1\right)-\frac{\mu}{2} \sum_{r} \sigma_{r}^{z}
$$

and:

$$
\varphi(r)=2 a^{\prime}>0 \text { for }|r|=1
$$

$$
\operatorname{Max}_{\alpha} \sum_{r:|r|>1}\left(\left|r_{\alpha}\right|+2\right)|\varphi(r)|<4 v a^{\prime} .
$$

The latter case is an Ising antiferromagnet. The appropriate $A_{A}, P_{A}$ and $Q_{\Lambda}$ for these models are defined by $(3.4,5)$ for the former and by $(3.7,8)$ for the latter. For fixed $t$, the geometrical properties of the contours are the same as in model (2). 
Let $G$ be a contour; we divide the set of its jumps into two classes:

(1) jumps occuring between some $G(t)$ and $G(t+1)$, and such that the jumping cube has a common face with $G(t)$. These we call $y$-jumps or superficial jumps.

(2) jumps occuring between some $G(t)$ and $G(t+1)$, and such that the jumping cube has no common face with $G(t)$. These we call $z$-jumps, or internal jumps.

If a jump is internal, all its faces are effective in $G(t+1)$. Contrary to the previous case, there is no symmetry between the two classes of jumps, and no relation between the class to which a jump belongs and the initial occupation state of the jumping cube.

We define $y(),. z(),. Y(),. Z($.$) , etc. as in Section (6). In the present$ case, $g(t)$ is not determined by $y($.$) and z($.$) . However:$

Therefore

$$
|g(t+1)-g(t)-2 v z(t)| \leqq 2 v y(t) .
$$

$$
|g(t+1)-g-2 v Z(t)| \leqq 2 v Y(t) .
$$

The periodicity condition $G(T+1)=G(1)$ implies that each jumping cube occurs an even number of times, and therefore at least twice. However, there is no general relation between the numbers of $y$-jumps and $z$-jumps using the same cube.

We now obtain the relevant estimates.

Lemma 10. The probability $P(G)$ of a contour $G$ satisfies:

$$
P(G) \leqq \exp \left[-\frac{c}{T} \sum_{t=1}^{T} g(t)\right]\left(\frac{h}{2 T}\right)^{X}
$$

where $c$ is given by (5.10) for the Ising ferromagnet, and by

$$
c=a^{\prime}-\frac{1}{4 v} \operatorname{Max}_{\alpha} \sum_{r:|r|>1}\left(\left|r_{\alpha}\right|+2\right)|\varphi(r)|-\frac{|\mu|}{2 v}
$$

for the Ising antiferromagnet.

Proof. The proof is identical with that of Lemma 6 for the ferromagnet. For the antiferromagnet and for nearest-neighbour-interaction, it is a straightforward transcription of the proof in [5]. The contribution of the tail of the potential has been estimated by the method of Ref. [7].

We also need an entropy estimate.

Lemma 11. The number of possible contour shapes with given $g \equiv g(1)$ and given $y($.$) and z($.$) is at most:$

$$
S(g, y(.), z(.)) \leqq 3^{g+v(Y+Z)-v} 2^{g / 2 v+2(Y+Z)} \prod_{t=1}^{T}[2 g(t)]^{y(t)}\left(Z^{\prime}(t)\right)^{z(t)} .
$$


The remark that follows Lemma 7 also applies to Lemma 11. The proof is almost identical with that of Lemma 9. There are at most $3^{g+v(Y+Z)-v} 2^{g / 2 v+Y+Z}$ ways to choose $G(1)$ with $g(1)=g$, and the $(Y+Z) / 2$ cubes that characterize the jumps. There are $\left(\begin{array}{c}Y+Z \\ Y\end{array}\right) \leqq 2^{Y+Z}$ ways to decide which of these cubes, counted twice, give rise to $y$-jumps and which to $z$-jumps. For fixed $G(t)$, the number of possible $y$-jumps is at most $2 g(t)$, and that of possible $z$-jumps is at most $Z^{\prime}(t)$. The lemma follows immediately.

We now formulate the result, after reintroducing the temperature by (4.10).

Theorem 4. The Ising model with transverse field, as defined by (3.3) and (5.1) or (7.1) exhibits a phase transition for all $\tilde{c}>0, \beta>\beta_{0}(\tilde{c})$ and $\tilde{h} \leqq \tilde{h}_{0}(\beta, \tilde{c}) \cdot \beta_{0}(\tilde{c})$ is an estimate of the classical type: $\beta_{0}(\tilde{c})=C t \tilde{c}^{-1} . \tilde{h}_{0}(\beta, \tilde{c})$ is a function of the form $\tilde{h}_{0}(\beta, \tilde{c})=\beta^{-1} h_{0}(c)$, where $h_{0}(c) \approx c^{\frac{1}{2}}$ for $c \rightarrow \infty$. In particular, when $\beta \rightarrow \infty$ for fixed $\tilde{c}, \tilde{h}_{0}(\beta, \tilde{c}) \approx \beta^{-\frac{1}{2}}$.

The shape of the two-phase region is indicated on Fig. 7 in the $(\tilde{h}, \beta)$ plane, for fixed $(\tilde{\varphi}, \tilde{\mu}, \tilde{c})$.

Proof. It is sufficient to prove (3.20). We collect the estimates from Lemmas 10 and 11 and obtain:

where:

$$
\pi(r) \leqq 3^{-v} \sum_{g \geqq 2 v}\left(\frac{g}{2 v}\right)^{\frac{v}{v-1}} 3^{g} 2^{g / 2 v} S(g)
$$

$$
\begin{aligned}
& S(g)=\sum_{y(.), z(.)} \operatorname{Sup}_{g(t), t \geqq 2} \exp \left[-\frac{c}{T} \sum_{t=1}^{T} g(t)\right] \\
& \cdot \prod_{t=1}^{T}\left[\frac{2 \alpha}{T} g(t)\right]^{y(t)}\left[\frac{\alpha}{T} Z^{\prime}(t)\right]^{z(t)}
\end{aligned}
$$

where now $\alpha=2 \cdot 3^{v} h$. The proof is a combination of those of Theorems 2 and 3 and will only be indicated briefly. We first perform the summation over the superficial $y$-jumps and take the Supremum over $g($.$) , thereby$ obtaining:

$$
\begin{aligned}
& S(g) \leqq \exp \left[-\frac{A(T)}{T} g\right] \sum_{z(.)} \exp \left[-\frac{2 v}{T} \sum_{t=1}^{T} z(t) A(T-t)\right] \\
& \cdot \prod_{t=1}^{T}\left[\frac{\alpha}{T} Z^{\prime}(t)\right]^{z(t)}
\end{aligned}
$$

where $A(0)=0$ and:

$$
A(t+1)=A(t)+c-2 \alpha \exp \left[2 v \frac{A(t)}{T}\right] .
$$


We then perform the sum over $z($.$) as in the previous section. Taking$ the limit $T \rightarrow \infty$, we obtain:

where:

$$
\pi(r) \leqq 3^{-v} \sum_{g \geqq 2 v}\left(\frac{g}{2 v}\right)^{\frac{v}{v-1}} 3^{g}(2 u)^{g / 2 v} v^{-1}
$$

$$
\begin{gathered}
u=\frac{2 \alpha}{c}+\left(1-\frac{2 \alpha}{c}\right) \exp (-2 v c) \\
v=1-\frac{2 \alpha^{2}}{c}-\frac{\alpha}{2 v c}\left(1-\frac{2 \alpha}{c}\right)[1-\exp (-2 v c)] .
\end{gathered}
$$

Theorem 4 follows immediately from $(7.13,14,15)$.

\section{Anisotropic Heisenberg Antiferromagnet}

The hamiltonian $H=K+U$ is defined by (3.1) and (7.1, 2, 3). The appropriate $A_{\Lambda}, P_{\Lambda}$ and $Q_{\Lambda}$ are defined by $(3.7,8)$. For fixed $t$, the geometrical properties of the contours are the same as in models (2) and (4). The possible jumps can be divided into $a$-jumps and $b$-jumps as in Section 3 . Clearly, the $b$-jumps are always superficial. Their relation with the contours to which they belong is the same as for model (2). On the contrary, the $a$-jumps involve pairs of adjacent cubes in the same situation (external vs. internal) with respect to the contours. We again divide the set of jumps into two classes.

(1) jumps from $G(t)$ to $G(t+1)$ such that one of the jumping cubes has a face in common with $G(t)$. These we call $y$-jumps or superficial jumps. This class includes all $b$-jumps.

(2) jumps from $G(t)$ to $G(t+1)$ such that the jumping cubes have no face in common with $G(t)$. These we call $z$-jumps or internal jumps.

We define $y(),. z($.$) , etc. as in Section 6. In the present case:$

$$
|g(t+1)-g(t)-(4 v-2) z(t)| \leqq(4 v-2) y(t) .
$$

Let $Y_{a}$ and $Y_{b}$ be the numbers of superficial $a$-jumps and $b$-jumps. Then, $X_{a}=Y_{a}+Z, X_{b}=Y_{b}$ and $X=X_{a}+X_{b}=Y+Z$.

We now obtain the relevant estimates.

Lemma 12. The probability $P(G)$ of a contour $G$ satisfies:

$$
P(G) \leqq \exp \left[-\frac{c}{T} \sum_{t=1}^{T} g(t)\right]\left(\frac{a}{T}\right)^{X_{a}}\left(\frac{b}{T}\right)^{X_{b}}
$$

where $c$ is given by (7.7).

Proof. The proof is identical with that of Lemma 10 in the antiferromagnetic case. 
We need also an entropy estimate.

Lemma 13. The number of possible contour shapes with given $g \equiv g(1)$, and given $y(),. z($.$) and Y_{b}$, is at most:

$$
\begin{aligned}
& S\left(g, y(.), z(.), Y_{b}\right) \leqq 3^{g-v+2 v X} 2^{g / 2 v+4 X} v^{Z}(2 v)^{Y_{a}} \\
& \cdot(2(v-1))^{Y_{b}}\left(\begin{array}{c}
Y \\
Y_{b}
\end{array}\right) \prod_{t=1}^{T}(g(t))^{y(t)}\left(Z^{\prime}(t)\right)^{z(t)} .
\end{aligned}
$$

The remark that follows Lemma 7 also applies to Lemma 13. The proof is almost identical with that of Lemma 11. There are at most $3^{g+2 v X-v}$ $\cdot 2^{g / 2 v+2 X}$ ways to choose $G(1)$ with $g(1)=g$ and the $X$ cubes that characterize the jumps. From the $2 X$ jumping cubes we now extract the $Z$ pairs that define the internal jumps. We can extract the first cube of a pair in $\left(\begin{array}{c}2 X \\ Z\end{array}\right)$ ways, the second in $(2 v)^{Z}$ ways, and each pair is counted twice. This gives a factor $\left(\begin{array}{c}2 X \\ Z\end{array}\right) v^{Z} \leqq 2^{2 X} v^{Z}$. We next choose, among the $Y$ times for $y$-jumps, those that correspond to $a$-jumps and $b$-jumps, thereby obtaining $\left(\begin{array}{l}Y \\ Y_{b}\end{array}\right)$. We finally choose $G(t)$ in the order of increasing $t$. From a given $G(t)$, the number of possible superficial $a$-jumps is at most $2 v g(t)$, and that of $b$-jumps $2(v-1) g(t)$. The number of possible $z$-jumps at time $t$ is $Z^{\prime}(t)$. Collecting all these estimates, we obtain (8.3). result.

We now reintroduce the temperature by (4.10) and formulate the

Theorem 5. The anisotropic Heisenberg antiferromagnet defined by (3.1), (7.1) exhibits a phase transition for all $\tilde{c}>0, \beta>\beta_{0}(\tilde{c})$ and $\tilde{\alpha} \leqq \tilde{\alpha}_{0}(\beta, \tilde{c})$. Here $c$ is defined by (7.7), $\beta_{0}(\tilde{c})=C t \tilde{c}^{-1} . \alpha$ is defined by:

$$
\alpha=2^{4} \cdot 3^{2 v}(v a+(v-1) b) .
$$

The function $\tilde{\alpha}_{0}(\beta, \tilde{c})$ tends to zero like $\beta^{-\frac{1}{2}}$ when $\beta$ tends to infinity for fixed $(\tilde{\varphi}, \tilde{\mu}, \tilde{c})$.

In the $(\tilde{\alpha}-\beta)$ plane, for fixed $(\tilde{\varphi}, \tilde{\mu}, \tilde{c})$, the two-phase region still has the shape indicated on Fig. 7.

Proof. It is sufficient to prove (3.20). Collecting the estimates from Lemmas 12 and 13 and performing the sum over $Y_{b}$, we obtain:

$$
\pi(r) \leqq 3^{-v} \sum_{g \geqq 2 v}\left(\frac{g}{2 v}\right)^{\frac{v}{v-1}} 3^{g} 2^{g / 2 v} S(g)
$$


where:

$$
S(g)=\sum_{y(\cdot), z(\cdot)} \operatorname{Sup}_{g(t), t \geqq 2} \exp \left[-\frac{c}{T} \sum_{t=1}^{T} g(t)\right] \prod_{t=1}^{T}\left(\frac{2 \alpha}{T} g(t)\right)^{y(t)}\left(\frac{\gamma}{T} Z^{\prime}(t)\right)^{z(t)}
$$

where:

$$
\gamma=2^{4} 3^{2 v} v a(\leqq \alpha)
$$

The end of the proof is identical with that of Theorem 4. The result, which implies Theorem 5, is:

$$
\pi(r) \leqq 3^{-v} \sum_{g \geqq 2 v}\left(\frac{g}{2 v}\right)^{\frac{v}{v-1}} 3^{g} 2^{g / 2 v} u^{g /(4 v-2)} v^{-1}
$$

where

$$
\begin{gathered}
u=\frac{2 \alpha}{c}+\left(1-\frac{2 \alpha}{c}\right) \exp [-(4 v-2) c] \\
v=1-\gamma\left\{\frac{2 \alpha}{c}+\left(1-\frac{2 \alpha}{c}\right) \frac{1-\exp [-(4 v-2) c]}{(4 v-2) c}\right\} .
\end{gathered}
$$

\section{Concluding Remarks}

We have proved that the phase transition which occurs in the Ising ferromagnet or antiferromagnet and in classical lattice gases with nearest-neighbour hard cores [5] is not destroyed if one adds a transverse magnetic field or a transverse interaction (or kinetic energy), provided the added term is sufficiently small. The method relies on Trotter's formula, and is thereby a perturbation method around the classical model. The transverse terms appear in the form of jumps that transform classical configurations between themselves. As regards the domain of validity of our proof, two situations emerge. In models (1) and (2), the jumps are superficial, and we can allow a ratio of transverse to longitudinal terms that tends to a non zero constant when $\beta$ tends to infinity. In models (3), (4) and (5), on the contrary, the jumps can be internal, and the allowed ratio of transverse to longitudinal terms tends to zero like $\beta^{-\frac{1}{2}}$ when $\beta$ tends to infinity.

We have restricted our attention to nearest-neighbour and nextto-nearest-neighbour transverse interaction. It is certainly possible to drop this restriction, but connectedness by edge will then no longer be the useful notion to define contours, and the entropy estimates will become more complicated.

Acknowledgements. I am grateful to D. Ruelle and H. Araki for helpful remarks. 


\title{
References
}

1. Peierls, R.: Proc. Cambridge Phil. Soc. 32, 477 (1936).

2. Griffiths, R.: Phys. Rev. 136 A, 437 (1964).

3. Dobrushin, R. L.: Theor. Prob. Appl. 10, 209 (1965).

4. - 5 th Symp. on Math. Stat. and Prob. Vol. III, p. 73, Univ. of Calif. Press (1967).

5. - Funct. Anal. Appl. 2, 44 (1968).

6. Berezin, F. A., and Ya. G. Sinai: Proc. Moscow. Math. Soc. 17, 197 (1967).

7. Ginibre, J., A. Grossmann, and D. Ruelle: Commun. Math. Phys. 3, 187 (1966).

8. Trotter, H.: Proc. Am. Math. Soc. 10, 545 (1959).

9. Mermin, N., and H. Wagner: Phys. Rev. Letters 17, 1133 (1966).

10. Fisher, M.: Rep. Prog. Phys. 30, 615 (1967).

11. Ruelle, D.: Helv. Phys. Acta 36, 789 (1963).

12. Ginibre, J.: Lecture given at the Colloque du C.N.R.S., Gif-sur-Yvette, May 1969.

\author{
J. Ginibre \\ Laboratoire de Physique \\ Théorique et Hautes Energies \\ Bâtiment 211 \\ Faculté des Sciences \\ F 91 Orsay
}

